\title{
Magnetic Properties of an $S=2$ Ladder Spin Model Applied to a New Quasi-One-Dimensional Magnet $\left[\mathrm{Mn}(\text { phen })_{3}\right](\mathrm{TCNQ})_{2} \cdot \mathrm{H}_{2} \mathrm{O}$
}

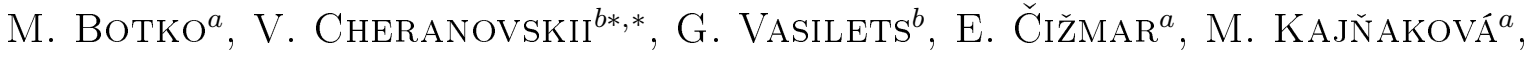 \\ A.G. Anders ${ }^{c}$, V. Starodub ${ }^{d}$ AND A. Feher ${ }^{a}$ \\ ${ }^{a}$ Institute of Physics, Faculty of Science, P.J. Šafárik University, Park Angelinum 9, 04154 Košice, Slovakia \\ ${ }^{b}$ Department of Chemistry, V.N. Karazin Kharkiv National University, Svobody Sq. 4, 61022 Kharkiv, Ukraine \\ ${ }^{c}$ Department of Physics, V.N. Karazin Kharkiv National University, Svobody Sq. 4, 61022 Kharkiv, Ukraine \\ ${ }^{d}$ Institute of Chemistry, Jan Kochanowski University of Humanities and Sciences, \\ Świętokrzyska 15G, 25-406 Kielce, Poland
}

\begin{abstract}
The effective spin $S=2$ Heisenberg ladder model with free-spin admixtures was proposed for the study of the low-temperature magnetic properties of the complex compound $\left[\mathrm{Mn}(\mathrm{phen})_{3}\right](\mathrm{TCNQ})_{2} \cdot \mathrm{H}_{2} \mathrm{O}$. The temperature dependence of magnetic susceptibility was found to be close to experimental data.
\end{abstract}

DOI: $10.12693 /$ APhysPolA.126.20

PACS: $75.50 . \mathrm{Xx}, 75.10 . \mathrm{Jm}, 76.10 . \mathrm{Pq}$

\section{Introduction}

Anion-radical salts of tetracyanoquinodimethane (TCNQ) containing transition metals complex cations are interesting objects due to their ability to form magnetically ordered materials [1]. One of them is $\left[\mathrm{Mn}(\mathrm{phen})_{3}\right](\mathrm{TCNQ})_{2} \cdot \mathrm{H}_{2} \mathrm{O}$. This compound was synthesized using procedure described in [2] for a similar $\mathrm{Fe}$ containing salt. The X-ray diffraction study of monocrystalline sample suggests a network of intermolecular carbon-nitrogen short bonds between paramagnetic cations $\left[\mathrm{Mn}(\mathrm{phen})_{3}\right]^{2+}(S=5 / 2)$ and TCNQ anion-radicals $(s=1 / 2)$. Thus, we distinguish linear sequences of spin moments of decorated ladder type, as shown schematically in Fig. 1.

This ladder may be described by the following Heisenberg spin Hamiltonian:

$$
\boldsymbol{H}=\sum_{i=1}^{N}\left[J_{1} \sum_{j=1}^{2} \boldsymbol{S}_{i, j}\left(\boldsymbol{s}_{i, 2 j-1}+\boldsymbol{s}_{i, 2 j}+\boldsymbol{s}_{i+1,2 j}\right)+J_{2} \boldsymbol{s}_{i, 2} \boldsymbol{s}_{i, 4}\right],
$$

where $\boldsymbol{S}_{i, j}$ is a $S=5 / 2$ spin operator that corresponds to $\mathrm{Mn}^{2+}$ cation and $s_{i, j}$ is a $s=1 / 2$ spin operator of the TCNQ anion; the first index $i$ enumerates lattice unit cells and the second index enumerates spins inside the unit cell. Analysis of structural data shows that the coupling parameters should be small and obey the condition $J_{2} \gg J_{1}$. According to the extended Lieb theorem [3], this spin ladder should have unique singlet ground state. However, the experimental temperature dependence of the magnetic susceptibility has paramagnetic behavior in

\footnotetext{
*corresponding author; e-mail: cheranovskii@i.ua
}

disagreement with the above model. To resolve this contradiction, we assume that the real material has some number of free structural units, $\left[\mathrm{Mn}(\mathrm{phen})_{3}\right](\mathrm{TCNQ})$, with the ground state spin $S=2$. In addition, due to the relationship between the coupling parameters of two TCNQ units and the cations of $\mathrm{Mn}^{+2}$, a singlet pair forms to suppress strongly the magnetic interactions between structural units. Consequently, a small energy gap exists between the singlet ground state of the salts and the magnetic excitations, thereby qualitatively explaining the magnetic data.

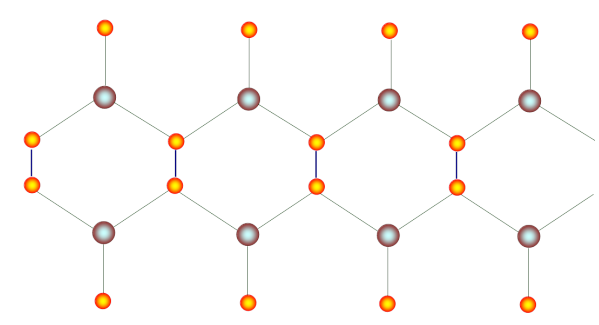

Fig. 1. Magnetic sublattice of the studied salt. Green and yellow balls correspond to $\left[\mathrm{Mn}(\text { phen })_{3}\right]^{2+}$ cations and TCNQ anions respectively.

\section{Spin ladder model}

In order to estimate the values of the effective interactions of the neighboring structural units, we performed an exact diagonalization study of the ground state and the first excited state energies of the lattice fragments, having two structural units connected by a TCNQ dimer along and perpendicular to the ladder. According to our calculations, we have the effective ferromagnetic coupling $J_{f}$ of the structural units along the ladder and an antiferromagnetic coupling $J_{a}$ perpendicular to the ladder. These quantities depend on the ratio of coupling param- 
eters $R=J_{2} / J_{1}$ of the Hamiltonian $\boldsymbol{H}$, and this dependence is shown in Fig. 2.

In the result we have the following approximate mapping of the Hamiltonian $\boldsymbol{H}$ onto a spin $\tilde{S}=2$ rectangular ladder model with antiferromagnetic interactions in legs and along diagonals, but with ferromagnetic interactions in rungs, such that:

$$
\begin{gathered}
\boldsymbol{H}_{e f f}=J_{a} \sum_{i=1}^{N}\left[\tilde{S}_{i, 1}\left(\tilde{S}_{i, 2}+\tilde{S}_{i+1,2}\right)+\tilde{S}_{i, 2} \tilde{S}_{i+1,1}\right]+ \\
J_{f} \sum_{i=1}^{N}\left[\tilde{S}_{i, 1} \tilde{S}_{i+1,1}+\tilde{S}_{i, 2} \tilde{S}_{i+1,2}\right] .
\end{gathered}
$$

It can be proven, similar to [3], that despite of diagonal interactions, this spin ladder has a nondegenerate singlet ground state.

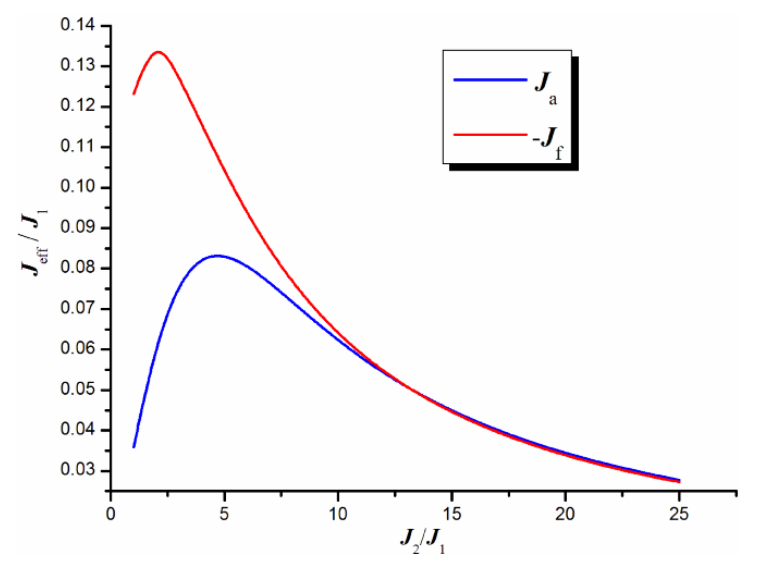

Fig. 2. The effective coupling constants vs $\mathrm{R}$.

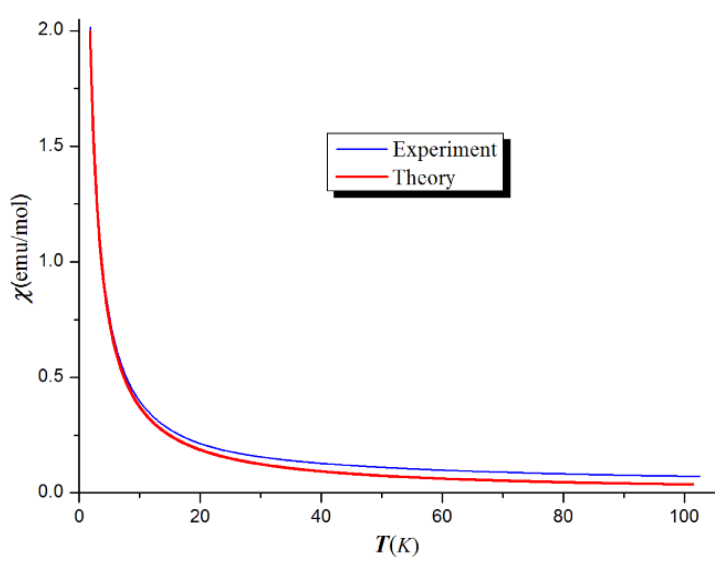

Fig. 3. Comparison of experimental and theoretical temperature dependencies of molar magnetic susceptibility of the salt.

\section{Numerical simulation for magnetic susceptibility}

Numerical calculations of the temperature dependence of magnetic susceptibility $\chi_{e f f}(T)$ for an effective spin ladder cluster with $N=3$, based on an exact diagonalization study, demonstrates behavior similar to the corresponding Ising ladder model. We use Ising approximation for the numerical estimation of $\chi_{\text {eff }}(T)$ for an infinite $S=2$ ladder by means of a standard transfer matrix technique.

The reason for the possible appearance of free structural units in the real material is similar to so-called 'broken chain effect' in other anion-radical salts of TCNQ $[4,5]$. The zero-field magnetic susceptibility of a structural unit $\chi_{\text {unit }}(T)$ has simple analytical form

$$
\chi_{\text {unit }}(T)=\frac{8 N_{A}\left(\mu_{B}\right)^{2}(5 \exp (x)+14)}{k_{B} T(5 \exp (x)+7)}, x=\frac{3 J_{1}}{k_{B} T}
$$

Here $N_{A}$ is Avogadro's number, $\mu_{B}$ is a Bohr magneton, and $k_{B}$ is the Boltzmann constant. For simplicity we put $g$-factors of $\mathrm{Mn}^{2+}$ and $\mathrm{TCNQ}^{-}$equal to 2, which does not contradict our ESR data.

Consequently, the total magnetic susceptibility per mole of $\mathrm{Mn}^{2+}$ cations was evaluated by the sum

$$
\chi_{\text {total }}(T)=(1-C) \chi_{\text {eff }}(T)+C \chi_{\text {unit }}(T),
$$

where $C$ is the molar amount of free structural units.

We performed calculations of $\chi_{\text {total }}(T)$ for different values of model parameters. One of the best fits to our experimental data in the temperature interval from $2 \mathrm{~K}$ to $100 \mathrm{~K}$ corresponds to the set of parameters: $J_{1}=0.2 \mathrm{~K}$, $J_{1}=1 \mathrm{~K}$ and $C=0.43$. The comparison of calculated dependence $\chi(T)$ and experimental data is given below in Fig. 2.

We also performed additional numerical study for ladder fragments with small single-ion anisotropy. We found that the behaviour of $\chi(T)$ is similar to isotropic model at $T>2 \mathrm{~K}$. Because of the absence of reliable experimental data for susceptibility below $2 \mathrm{~K}$, we restricted our analysis by minimal isotropic Heisenberg spin model.

\section{Acknowledgments}

This work was supported by the SAS Centre of Excellence CFNT MVEP, by the ERDF EU grant under the contract No. ITMS26220120047, and by the Slovak Research and Development Agency under the contract APVV-0132-11. The financial support of U. S. Steel Košice is gratefully acknowledged.

\section{References}

[1] L. Ballester, A. Guttiéres, M.F. Perpiñán, M.T. Azcondo, Coord. Chem. Rev 190-192, 447 (1999).

[2] C. Alonso, L. Ballester, A. Gutiérrez, M.F. Perpiñán, A.E. Sanchez, M.T. Azcondo, Eur. J. Inorg. Chem. 2005, 486 (2005).

[3] D.J. Klein, J. Chem. Phys. 77, 3098 (1982).

[4] Y. Liu, J.E. Drumheller, R.D. Willett, Phys. Rev. B 52, 15327 (1995).

[5] M.Botko, V.O. Cheranovskii, O.N. Kazheva, G.V. Shilov, O.A. Dyachenko, A.B. Verkin, M. Kucmin, V.A. Starodub, A. Radváková, M. Kajňaková, A. Fehera, Solid State Sciences 24, 85 (2013). 